Conclusion Not using a condom in serving customers in women indirect sex workers be risk factors for STIs. Need an effort to monitor the prevalence of sexually transmitted infections continued cooperation and support of stakeholder in pressing the prevalence of STIs

\section{P3.237 AN ASSESSMENT OF STD SCREENING PRACTICES IN NEW PATIENTS ATTENDING AN HIV PRIMARY CARE CLINIC}

doi:10.1136/sextrans-2013-051184.0694

R Blanchard, J Keller, A Wilkin, L Bachmann. Wake Forest University Health Sciences, Winston-Salem, NC, United States

Background Since 2003, national guidelines recommend that all new HIV patients undergo screening for sexually transmitted diseases (STD) during their initial visit. We evaluated STD screening practises in new patients attending an HIV Primary Care Clinic in North Carolina.

Methods This retrospective cohort study included HIV-infected patients greater than 18 years of age enrolled in the clinic between January 1, 2008 and December 31, 2010. Data on rapid plasma reagin (RPR) testing for syphilis and Neisseria gonorrheoeae (GC) and Chlamydia trachomatis (CT) testing by site of exposure, within 1 year of the new patient visit, were extracted via chart review to examine comprehensiveness of STD screening practises.

Results Between 2008 and 2010, 693 (68\% male and 32\% female) new patients entered care, most of whom were screened for syphilis within one year of the new patient visit $(94.5 \%$ in $2008,96.6 \%$ in $2009,96.9 \%$ in $2010, p=0.41)$. In contrast, only $21.3 \%$ were tested for GC and CT within one year of the first visit in 2008, $34.3 \%$ in 2009 , and $54.3 \%$ in 2010 ( $p=<0.0001$ ). Of those screened, the prevalence of past/current syphilis ranged from $8.4-10.1 \%(p=0.81)$ while positive testing for either GC or CT ranged from $2.3-9.1 \%$ $(p=0.20)$. In 2008, 1 rectal and 1 pharyngeal GC and CT test was performed versus 9 rectal and 8 pharyngeal tests in 2009 and 20 rectal and 14 pharyngeal tests in 2010. Of these, 13.3\% of rectal tests and $8.7 \%$ of pharyngeal tests were positive, in contrast to $3.7 \%$ of genital tests.

Conclusion While screening practises improved from 2008 to 2010, new HIV patients continue to be under-screened for gonorrhoea and chlamydia, particularly at extra-genital sites. This deficiency is particularly important as, of those screened, prevalence was higher in extra-genital compared to genital sites. Efforts should be made to increase routine extra-genital screening.

\section{P3.238 GENERATING EVIDENCE THROUGH SEROSURVEILLANCE; HELPING IN PROGRAMMIE DESIGNING TO MITIGATE SEXUALLY TRANSMITTED INFECTIONS (STIS) AMONG FEMALE SEX WORKERS (FSWS) IN BANGLADESH}

doi:10.1136/sextrans-2013-051184.0695

'M Amin, ${ }^{2}$ M A Rahman, 'A F Khuda, 'S Rasin, 'L Rahman, 'F Sultana, 'M S Islam. 'Save the Children, Dhaka, Bangladesh; 'National AIDS/STD Programme, Dhaka, Bangladesh

Background The overall prevalence of HIV and active syphilis are $0.7 \%$ and $3 \%$ respectively revealed in 9th serosurveillance among most at risk population. High active syphilis rates suggest practise of unsafe sex and a surrogate marker of unsafe sex is active syphilis. STIs and HIV are linked as they share the same risk behaviour, STIs facilitate acquisition and transmission of HIV, and some STI pathogens become more virulent in presence of HIV-related immunodeficiency. Under Global Fund support and initiative Save the Children stepped in (2008) with essential service (primly STI management, Condom distribution, BCC session through DICs and outreach) for FSWs nationwide in 51 districts which includes all serosurveillance sampling sites for FSWs.
Methods 8th round serosurveillance was conducted between July and December 2007 where total 4797 FSWs in 15 cities were sampled. 9th serosurveillance was conducted between December 2010 to June 2011 where total 3568 FSWs were sampled from 13 cities of Bangladesh. Same sampling methodology followed in both the rounds. Sero-surveillance sampling site were under implementation coverage.

Results In 8th sero-surveillance, $>5 \%$ active syphilis was detected in five sites namely street FSWs of Chittagong, Rangpur, Dhaka, hotels FSWs of Sylhet (8.3\%) and casual FSWs in Chandpur. In 9th serosurveillance, $>5 \%$ active syphilis was detected in three sites, street FSWs of Hili, Chittagong and hotel FSWs of Sylhet. Active Syphilis rate decreased in all the 5 sites except hotel FSWs in Sylhet (9.3\%). Moreover, there is decreasing trends of active syphilis among FSWs in several sites in 9th round comparing to 8th.

Conclusion High active syphilis rate highlight the need for ongoing programme intensification to decline STI trend. Evidence with good programmatic implication extrapolated and translated through serosurveillance could tailor-made the ongoing intervention and also designing the future programme.

\section{P3.239 HIV AND SYPHILIS INFECTION AND RISK BEHAVIOURS AMONG FEMALE SEX WORKERS IN PARAGUAY IN 2012}

doi:10.1136/sextrans-2013-051184.0696

'G Aguilar, ${ }^{2} \mathrm{~J}$ Jacobson, 'L Gimenez, ${ }^{1} \mathrm{~A}$ Barboza, ${ }^{1} \mathrm{~A}$ Kawabata. ${ }^{1}$ National Program of Control of HIVAidsSTI, Asuncion, Paraguay, ${ }^{2}$ Independent Consultant, Bogota, Colombia

Introduction Syphilis is an important public health problem in Paraguay. A 2006 study found high levels of syphilis (19.0\%) infection and unprotected sex with most recent client (also 19.0\%) and moderate level of HIV infection (1.8\%) in female sex workers (FSW). Several HIV and STI prevention interventions have been put in place since then. A recent study sought to assess current infection and risk behaviours.

Methods 432 FSW were recruited from sex work locations in the greater metropolitan area of Asuncion using time-location sampling between January and May, 2012. A face-to-face survey and rapid and confirmatory biological tests in accordance with national algorithms assessed risk behaviours and syphilis and HIV infections. Weighted statistical analysis accounted for the sampling strategy and adjusted for clustering by recruitment venue. Adjusted $95 \%$ confidence intervals (CI's) for the 2006 and 2012 studies were compared to identify significant trends.

Results Median age of participants was 25 years (interquartile range (IOR), 21-32). Median age of first sex work was 20 years (IOR 18-24). Differences in VIH prevalence among 2006 (1.8\%) and 2012 $(2.5 \% \%)$ studies weren't significant $(P=0.4)$. Prevalence syphilis $(25.3 \%$, IC $21.3 \%-29.8 \%)$ were higher than 2006 estimates $(P<0.05))$. Median number of clients during the past week was 15 (IOR 8-25). 40.5\% (CI 35.8-45.3) reported drinking at last sex with a client. Condom use with last client was $98.1 \%$ (IC 96.2\%-99.1\%), increased over $2006(P<0.05)$ Among 59.3\% who had a stable partner, $77.3 \%$ (IC 71.4-82.0) had not used a condom at last sex with a stable partner.

Conclusion Increasing levels of syphilis infection suggest a need to reevaluate and intensify STI prevention and control interventions, with greater emphasis on diagnosis and treatment, reducing drinking with clients as well as providing alternatives to sex work for FSW.

\section{P3.240 DOES THE PREVALENCE OF SEXUALLY TRANSMITTED DISEASES ADEQUATELY REFLECT SEXUAL TRANSMISSION OF HEPATITIS C IN THE HIV-INFECTED POPULATION?}

doi:10.1136/sextrans-2013-051184.0697 
'G V Escota, ${ }^{2}$ T Taniguchi, 'B P Stoner, ${ }^{1} \mathrm{~N}$ F Onen. 'Washington University School of Medicine, Division of Infectious Diseases, Saint Louis, MO, United States; ${ }^{2}$ Chiba University Graduate School of Medicine, Department of Developmental Genetics, Chiba, Japan

Background Recent data suggest sexual transmission of hepatitis $\mathrm{C}$ virus (HCV). However, data on the association between HCV and sexually transmitted disease (STD) prevalence are limited.

Methods This was a retrospective cohort study of treatment-naïve HIV-infected adults $\geq 18$ years first engaging at Washington University HIV Clinic from 2001 to 2009, who had routine STD and HCV antibody testing done. Gonorrhea, chlamydia, syphilis, and HCV cases were defined by positive urine nucleic acid amplification test for Neisseria gonorrhoeae, Chlamydia trachomatis, reactive serum rapid plasma reagin, and positive HCV antibody, respectively. Associations with HCV and STD using $\chi 2$, Student's t, and Wilcoxon tests were determined.

Discussion Of 926 subjects (median age 32 years, 70\% African American, 44\% heterosexual, 42\% men-who-have-sex-with-men [MSM], 4\% injection drug users [IDU]), 8\% had HCV (range $5-11 \%$ /year). Baseline STD was prevalent in $27 \%$ (18-34\%/year). The prevalence of gonorrhoea, chlamydia and syphilis were $12 \%$ (7-21\%/year), 12\% (6-17\%/year) and 10\% (5-16\%/year), respectively. Subjects with HCV were older (42 years, interquartile range [IOR 38-48] versus 31 years, [IOR 24-40]) $(p<0.001)$ and more likely to report past IDU $(30 \%$ versus $2 \%)(p<0.001)$ than those without. Male subjects with HCV were less likely to be MSM (28\% vs $66 \%)(p<0.001)$ and $36 \%$ of subjects with HCV were heterosexuals without past IDU. Subjects with HCV were less likely to have STD $(17 \%$ vs $28 \%, p=0.06)$, although this finding did not reach statistical significance. Furthermore, the number and type of STDs at presentation were not associated with prevalent HCV.

Conclusion Hepatitis C was prevalent in approximately 1 in 10 persons engaging in HIV outpatient care over nine years. A high prevalence of HCV among heterosexuals without past IDU suggests a possible role for sexual transmission of HCV not reflected by STD prevalence. Continued universal HCV screening among HIVinfected adults is imperative.

\section{P3.241 EPIDEMIOLOGY OF SEXUALLY TRANSMITTED INFECTIONS IN EUROPE 1990-2011}

doi:10.1136/sextrans-2013-051184.0698

G Spiteri, M van de Laar. European Centre for Disease Prevention and Control, Stockholm, Sweden

Background STI are a significant cause of morbidity in Europe, particularly among young adults and men who have sex with men (MSM). Monitoring the spread of STI across key populations allows development of targeted prevention interventions.

Methods Surveillance for chlamydia, gonorrhoea and syphilis in the European Union (EU/EEA) is coordinated by the European Centre for Disease Prevention and Control since 2009. Data reported by Member States covering the period 1990-2011 were analysed.

Results In 2011, 346911 cases of chlamydia (rate: 175 per 100000 population) were reported compared to 39179 cases of gonorrhoea (12.6/100000) and 19798 cases of syphilis (4.9/100000). Chlamydia was more frequently reported among women (male-to-female ratio: 0.7 ) in contrast to gonorrhoea and syphilis (male-to-female ratios: 2.7 and 3.9 respectively). The highest age and gender-specific rates were observed among 15-19 year old females for chlamydia (1748); 20-24 year-old males for gonorrhoea (71) and 25-24 year-old males for syphilis (16.1). Young adults (15-24 years) accounted for $73 \%$ of chlamydia, $42 \%$ of gonorrhoea and $16 \%$ of syphilis cases. Transmission among MSM was more frequently reported for syphilis (42\%) and gonorrhoea (33\%) cases than for chlamydia (5\%). HIV co-infection was reported among $11 \%$ of gonorrhoea and $28 \%$ of syphilis cases. Rates of chlamydia have increased by $133 \%$ between 2000 and 2011 among countries reporting consistently. Gonorrhoea rates have increased by $27 \%$ since 2008 and syphilis rates increased by $10 \%$ in 2011.

Conclusion Surveillance data shows diversity in reported rates and trends across the EU/EEA. Increasing rates of chlamydia reflect strengthened case detection and improved diagnostics. Young adults constitute a large proportion of cases of chlamydia and gonorrhoea. Transmission among MSM accounts for the majority of syphilis and, increasingly, gonorrhoea spread. Both key populations need to be better targeted through specific prevention and control measures.

\section{P3.242 WITHDRAWN BY AUTHOR}

\section{P3.243 INFLUENCE OF SCALE AND ZONE ON SYPHILIS TREND INTERPRETATION}

doi:10.1136/sextrans-2013-051184.0699

1.2V Escamilla, ${ }^{3} \mathrm{~K}$ Hampton, ${ }^{3,4,1 \mathrm{P}}$ A Leone, ${ }^{1,3} \mathrm{~W}$ C Miller. 'Division of Infectious Diseases, Department of Medicine, University of North Carolina, Chapel Hill, NC, United States; ${ }^{2}$ Carolina Population Center, University of North Carolina, Chapel Hill, NC, United States; ${ }^{3}$ Department of Epidemiology, Gillings School of Global Public Health, University of North Carolina, Chapel Hill, NC, United States; ${ }^{4}$ HIV/STD Prevention and Care Branch, North Carolina Division of Public Health, Raleigh, NC, United States

Background We investigated spatial clustering of primary and secondary syphilis rates in North Carolina (2003-2010) using multiple scales and geographical boundaries. We examined the influence of changes in scale and boundary on identification of spatial clusters using two cluster detection methods, local Moran's I and Kulldorff's space scan statistic.

Methods We used two cluster detection methods: (1) local Moran's I with empirical Bayes (EB) standardised rate statistic and (2) Kulldorff's space scan statistic using a variable size moving circular window. We evaluated three geographic zones with decreasing boundary area, North Carolina, Piedmont region, and Mecklenburg County, at two spatial scales (census tract, census block group). We report results for Mecklenburg County.

Results Using local Moran's I, block group clusters were in the same location as tract clusters, but were more concentrated. Median rates were higher among block group clusters compared with tract clusters. As boundary areas decreased, some clusters in peripheral tracts and block groups were lost. High rate block groups were more likely to persist, while some high rate peripheral tracts were lost.

With Kuldorff's scan statistic, block group clusters were more concentrated than clusters in census tracts. Reducing boundary areas had little effect on census tract clusters detected using Kuldorff's scan statistic. Cluster size decreased significantly when the boundary was restricted to Mecklenburg County. The reduction in cluster size reflected loss of a few high rate block groups peripherally and many block groups with a rate of zero.

Clusters detected using local Moran's I and Kuldorff's scan statistic overlapped, but Kuldorff's scan clusters were much larger with a high proportion of zero rate tracts/block groups.

Conclusion In efforts to understand STI epidemiology spatially, investigators must carefully consider the spatial scale, geographical area of interest, and cluster detection approach.

\section{P3.244 PREVALENCE OF SEXUALLY TRANSMITTED INFECTIONS IN MULTIPLE SAMPLE TYPES COLLECTED FROM HIV-1 POSITIVE MEN}

doi:10.1136/sextrans-2013-051184.0700

${ }^{1} \mathrm{~F}$ Zhang, ${ }^{2} \mathrm{~S}$ R Morris, ${ }^{3} \mathrm{~J}$ P McGowan, ${ }^{2} \mathrm{~J}$ Milam, ${ }^{4} \mathrm{M}$ Dube, ${ }^{5} \mathrm{E} S \mathrm{Daar},{ }^{3} \mathrm{D}$ Dubson, ${ }^{6} \mathrm{C} S$ Hill, ${ }^{2} \mathrm{R}$ H Haubrich, ${ }^{1,7}$ C C Ginocchio. ${ }^{1}$ North Shore-LIJ Health System Laboratories, Lake Success, NY, United States; 'niversity of California, San Diego, CA, United States, 